\title{
Meta
}

Journal des traducteurs

Translators' Journal

\section{Alonso ArAguás, Icíar, PÁEz Rodríguez, Alba y SAMANiEgo SASTRE, Mario, eds. (2015): Traducción y representaciones del conflicto desde España y América. Una perspectiva interdisciplinar. Salamanca/Temuco: Ediciones Universidad de Salamanca/Universidad Católica de Temuco, 257 p.}

\section{$M \varepsilon T A$}

\section{Anne-Marie Gagné}

Volume 62, numéro 3, décembre 2017

URI : https://id.erudit.org/iderudit/1043959ar

DOI : https://doi.org/10.7202/1043959ar

Aller au sommaire du numéro

Éditeur(s)

Les Presses de l’Université de Montréal

ISSN

0026-0452 (imprimé)

1492-1421 (numérique)

Découvrir la revue

Citer ce compte rendu

Gagné, A.-M. (2017). Compte rendu de [ALONSO ARAGUÁs, Icíar, PÁEZ RODRÍGUEZ, Alba y SAMANIEGo SASTRE, Mario, eds. (2015): Traducción y representaciones del conflicto desde España y América. Una perspectiva interdisciplinar. Salamanca/Temuco: Ediciones Universidad de Salamanca/Universidad Católica de Temuco, 257 p.] Meta, 62(3), 655-657. https://doi.org/10.7202/1043959ar d'utilisation que vous pouvez consulter en ligne. 
science and education of the present day, which includes two essays: one by Maria Amalia Barchiesi, Apuntes para una semiótica de los textos cientificos divulgativos en lengua española, the other by Marco Paone and Lorena Paz López, Ciencia, ironía y traducción: Tiempo de silencio de Luis Martín-Santos. The first analyzes contemporary Spanish texts on ecology, focusing on the threatened Mexican oak tree producing the world's largest acorn. The analysis is taken from a semiotic point of view, drawing on such authorities as the semioticians Greimas, Jacobi and Latour and the work of those involved in popularizing science, the techniques used being presented as a form of translation: a fresh look at a well-known theme. The second is a study of the translation of scientific terms from a 1960s Spanish novel translated into French and Italian, an example of 'professional substratum fiction' ${ }^{1}$ much studied in France. This chapter is well illustrated with contrasting examples of translating strategies:

The final section focuses on image in scientific translation and consists of the texts by Francesc Rodríguez and Cecilio Garriga, Ilustraciones de la ciencia en la lexicografía española, and Peter Mason's El rol de las Canarias en la traducción visual del Nuevo Mundo para el Viejo Continente. The first is a very complete account (within the scope of a single chapter) of the role of illustrations in monolingual dictionaries and an in-depth study of those in the Spanish adaptation of the ground-breaking Petit Larousse illustré, in the early part of the twentieth century, appropriately profusely illustrated. The second chapter traces the many illustrations of the miraculous water-bearing garoé tree on the Canary Islands in Girolamo Benzoni's 1565 description (featuring in a book on the Americas, thus the title), its re-editions and many translations and the reinterpretations they both embodied and prompted.

As the editor rightly points out, the history of scientific translation is by definition an interdisciplinary endeavour. Not all of the contributors are primarily researchers in translation studies, which can lead to a wish for more depth in translation analysis. For example, in several texts the relations between translation and equivalence are hinted at rather than made explicit: to what extent did the translator have recourse to a pre-existing term in the target language, or did this new term have to be coined by the translator? But works such as these open up a dialogue where such issues can be investigated.

Translation specialists as well as those with an interest in the history of science and ideas will find this a rich repository.

\section{NOTE}

1. For more information on 'FASP,' see Petit, Michel and IsanI, Shaeda, eds. (2004): Aspects de la fiction à substrat professionnel. Bordeaux: Université Victor Segalen.

\section{REFERENCES}

De Beni, Matteo, ed. (2015 ): De los descubrimientos a las taxonomías. La botánica y la zoología en la lengua española del Renacimiento a la Ilustración. Mantova: Universitas Studiorum.

Ducos, Joëlle, ed. (2011): Sciences et langues au Moyen-Âge. Heidelberg: Winter.

Duris, Pascal, ed. (2008): Traduire la science. Hier et aujourd'hui. Pessac: Publications de la Maison des sciences de l'homme d'Aquitaine.

Alonso Araguás, Icíar, PÁez Rodríguez, Alba y Samaniego Sastre, Mario, eds. (2015): Traducción y representaciones del conflicto desde España y América. Una perspectiva interdisciplinar. Salamanca/Temuco: Ediciones Universidad de Salamanca/Universidad Católica de Temuco, $257 \mathrm{p}$.

Si la traducción ha sido frecuentemente conceptualizada en relación con el conflicto (y eso desde mucho antes de la formación de nuestra disciplina, por ejemplo, en Steiner 1975), el interés por su papel mediador está íntimamente vinculado con el surgimiento del traductor como objeto de estudio, a partir del Cultural Turn. La presente obra constituye un aporte significativo a ambos temas, proponiendo unas miradas cruzadas (provenientes de España y de América) sobre el conflicto $y$, ante todo, sobre su mediación intercultural e interlingüística.

Publicado por las Ediciones de la Universidad de Salamanca y por las de la Universidad Católica de Temuco, Traducción y representaciones del conflicto desde España y América. Una perspectiva interdisciplinar ha sido coeditado por Icíar Alonso Araguás, Alba Páez Rodríguez y Mario Samaniego Sastre. Presenta, a través de 14 artículos (incluyendo el último que desempeña la función de epílogo), las reflexiones que surgieron del encuentro Mediación, migraciones e interculturalidad. Perspectivas históricas y contemporáneas desde España y América que tuvo lugar en Salamanca, en febrero 2014. Convocado por la Universidad de Salamanca (USAL), este evento reunió a 3 grupos de investigación (Alfaqueque y GIR Traducción, Ideología y Cultura de la USAL, Frontera de Lenguas de la Universidad Católica de Temuco), así como a investigadores procedentes de varias otras instituciones. Cabe mencionar que la presente obra, también continúa en la línea del encuentro 
internacional sobre la historia lingüístico-cultural, realizado en Temuco en 2010, y del libro $L a$ mediación en tiempos de guerra: cruce de miradas desde España y América (Payàs y Zavala 2012), que resultó de este evento.

Al igual que estos últimos, este libro trata primero el conflicto desde una perspectiva histórica (partes I y II). Sin embargo, se amplió aquí el tema para abarcar también puntos de vista sobre temas contemporáneos (partes III y IV), entre los cuales, los medios de comunicación y las migraciones. Aunque algunos artículos abordan el «conflicto» como enfrentamiento armado (principalmente en las partes I y II) e incluso uno se enfoca precisamente en esta cuestión (p. 157), en general, la visión adoptada en el libro aquí reseñado se asemeja a la acepción más amplia del concepto propuesta por Mona Baker: "[...] a situaction in which two or more parties seek to undermine each other because they have incompatible goals, competing interests, or fundamentally different values» (2006: 9). Sin embargo, los autores comparten una visión que va más allá de una «incompatibilidad» y consideran también el carácter fundamentalmente asimétrico de estas situaciones. Finalmente, según los editores, la traducción oral y escrita se encuentra precisamente en estos espacios de conflicto-mediación donde se «establece la distancia necesaria para reconocer la alteridad en toda su amplitud» (p. 11-12).

La primera parte, «Traducción y conflicto en la frontera: Miradas desde América», comprende tres artículos. La destacable contribución de Gertrudis Payàs Puigarnau «Acercamiento a las dinámicas interétnicas hispano-mapuches en el paso de Colonia a República desde la historia disciplinar», examina las funciones lingüísticas e extralingüísticas desempeñadas durante el periodo por los intérpretes - funciones que incluyen lo que ella llama «administrar las distancias» entre los grupos étnicos - y como se instrumentaliza la interpretación. «Los parlamentos hispanomapuches como espacios de reconocimiento del Otro enemigo: huellas históricas, lingüísticas y territoriales», un artículo fundamentalmente pluridisciplinar de José Manuel Zavala Cepeda, Tom Dillehay, Gertrudis Payàs Puigarnau y Fabien Le Bonniec, propone analizar las huellas escritas de los parlamentos a través dos puntos de vista: arqueológico-etnohistórico-geográfico e etnohistóricolingüístico-traductológico. Por último, Florencia Roulet presenta en "Capitanes de amigos en la frontera de Mendoza: los usos indígenas de una institución colonial», el carácter ambiguo e híbrido de esta figura, así como de los múltiples papeles que cumple como mediador, protector y portavoz.

La segunda parte, «Miradas desde España y América», es igualmente constituida por tres artí- culos. «La mediación en la frontera canario-africana en los siglos XV y XVI: el resurgir de Adalid», de Marcos Sarmiento Pérez, describe el perfil del Adalid en esta época y sus prácticas mediadoras en el espacio fronterizo cristiano-musulmán, prácticas que se caracterizan por una mezcla de funciones, tales como guía, centinela, interprete lingüístico y cultural y negociador. En «Garcia de Orta: notas sobre las fronteras de la ciencia renacentista», Isabel Soler y Juan Pimentel presentan la trayectoria del médico portugués García de Orta, considerado como passeur culturel y su obra políglota, entendida como transfronteriza. Otra contribución a la historia de la interpretación, «Un intérprete en la geopolítica del imperio español en el Pacífico sur a finales del siglo XVIII: Máximo Rodríguez en Tahití» de Jesús Baigorri Jalón, explora la trayectoria de Rodríguez principalmente à través de su diario. Gracias a su predisposición para el aprendizaje de la lengua tahitiana, el intérprete termina ejerciendo no solamente como mediador cultural y lingüístico, sino también como diplomático, topógrafo, curandero y agricultor.

En la tercera parte de esta recopilación, «Representaciones interculturales en los medios de comunicación», Páez Rodríguez presenta un caso que pretende de "traducción intralingüística». En su artículo, «Una aproximación narrativa a las traducciones intralingüísticas del conflicto Repsol-YPF en la prensa española y argentina», sostiene que por motivos ideológicos-políticos, las producciones mediáticas de estos dos países crean distintas narrativas, las cuales influyen sobre el lector y su percepción del conflicto. Cristina Vidal Sales, por su parte, estudia en «Familiarmente extraños: construcción de la alteridad latinoamericana en el doblaje español» la representación de la latinoamericanidad a través del doblaje español de series estadounidenses, $\mathrm{o}$ "como se retraducen identidades ya traducidas» (p. 154). Demuestra que, en el doblaje, los estereotipos acerca de los hispanohablantes de la versión original están desplazados para corresponder a los arquetipos del «Otro» de la audiencia destinataria. En el último artículo que conforma esta parte, «El intérprete en los conflictos bélicos contemporáneos: identidades ambiguas en la prensa escrita» Icíar Alonso Araguás examina cómo se representan de diferentes maneras los intérpretes que trabajan en zonas de conflicto violentos (su nivel de visibilidad o de anonimato, su posición ambivalente en relación con ambos bandos) en los periódicos y los cuestionamientos éticos suscitados por estas representaciones.

La última parte «Dinámicas migracionales, interculturalidad y ciudadanía» presenta la contribución de Mario Samaniego Sastre, «Derivas de la diversidad: comunicación, espacio público político y frontera». A partir del concepto de diversidad y 
de sus vínculos con la intersubjetividad, el autor reflexiona sobre el espacio público-político, la comunicación oral-escrita y la frontera en las relaciones hispano-mapuches, con fin de repensar la convivencia en el mundo actual. El artículo de Francisco Raga Gimeno «En el corazón de la cultura. Hibridación y mediación intercultural en el ámbito de la salud mental», aborda el concepto de interculturalidad y sus vínculos con la interpretación en el campo psiquiátrico. Subraya el carácter cultural de este mismo y sugiere implantar lo que llama «espacios interculturales más integrales». El artículo de María López Ponz «From la otra orilla and back: representaciones del mestizaje en las traducciones al español de literatura hispanoestadounidense» trata de las estrategias utilizadas, en las traducciones españolas de obras de escritoras hispano-estadounidenses, para representar las diferentes formas de spanglish y los elementos biculturales y de cómo la posición y el habitus de los traductores influye en este proceso. Juan Jesús Zaro propone en su trabajo "La traducción de Shakespeare en la América de lengua española: entre la tradición y la transculturación», explorar las diferentes traducciones en español del autor a partir de una clasificación que distingue las traducciones tradicionales, de las transculturadas. Finalmente, el epílogo de la presente obra está constituido por la contribución de Yolanda Onghena «De contexto a contexto: movilidades transnacionales» que aborda la tensión identidaddiferencia en los contextos migratorios y propone aceptar la naturaleza dinámica del proceso identificación-diferenciación.

En resumen, Traducción y representaciones del conflicto desde España y América. Una perspectiva interdisciplinar propone un recorrido histórico polifacético de algunas prácticas e instituciones de mediación intercultural e interlingüísticas, que existieron a partir del siglo IV hasta la actualidad. La fuerza de esta recopilación radica, en nuestra opinión, en su planteamiento interdisciplinar que permite aprehender este mismo objeto a partir de las perspectivas de campos tan variados como la traductología, la historia, la antropología, la filosofía y la literatura. Esta aproximación innovadora presenta un panorama amplio y nos permite apreciar las distintas manifestaciones del fenómeno conflicto-mediación en toda su complejidad.

Sin que la calidad de las reflexiones expuestas se viera afectada, no podemos obviar que son las miradas españolas las que siguen dominando y que las miradas americanas se originan exclusivamente desde Chile y Argentina, y eso a pesar de la crítica del etnocentrismo que atraviesa todo el libro. Si, por una parte, el lugar en que se llevó al cabo el seminario del cual deriva este libro y el lugar de procedencias de los tres grupos involucrados ofrece una explicación satisfactoria, confiamos en que la inclusión de otras voces marginadas hubiera encajado perfectamente con el planteamiento de este proyecto y enriquecido todavía más el horizonte presentado.

Finalmente, el eclecticismo de los artículos, que resulta no precisamente del enfoque multidisciplinario, sino de la multiplicidad de los objetos, subtemas y perspectivas, así como del amplio periodo cubierto, constituye a la vez parte de la originalidad de la presente obra y su límite. Sin embargo, el hecho que se puede adquirir el ebook por capítulos no dejará de atraer académicos interesados solamente en uno $\mathrm{u}$ otro de los subtemas desarrollados.

\section{Anne-Marie Gagné Université de Montréal, Montreal, Canadá}

\section{REFERENCIAS}

BAker, Mona (2006): Translation and Conflict. A Narrative Account. Londres/Nueva York: Routledge.

Steiner, George (1975): After Babel. Oxford: Oxford University Press.

CAstro Ramírez, Nayelli, dir. (2013): Traducción, identidad y nacionalismo en Latinoamérica. Mexico: Bonilla Artiga, 286 p.

Le tournant de 2014 a été faste pour les publications sur l'histoire de la traduction en Amérique hispanique. En octobre 2013, le premier Diccionario histórico de la traducción en Hispanoamérica voit le jour en Espagne, comme suite logique et attendue du Diccionario histórico de la traducción en España (2009), tous deux coordonnés par Francisco Lafarga et Luis Pegenaute ${ }^{1}$. Au Mexique, en juin 2014², paraît Traducción, identidad y nacionalismo en Latinoamérica, ouvrage collectif dirigé par Nayelli Castro Ramírez, que nous abordons ici. Malgré le titre inclusif, qui autoriserait la présence de contributions sur le Brésil, l'ouvrage ne compte que des chapitres sur des sujets hispano-américains. Néanmoins, cette publication constitue, à n'en pas douter, un premier et louable effort d'historiciser la traduction de manière continentale, selon plusieurs axes. Ce texte est d'ores et déjà inclus dans la bibliographie de cours de traduction en Amérique hispanique . $^{3}$

La comparaison entre les perspectives latinoaméricaines $^{4}$ de facture mexicaine et le Dictionnaire historique de facture espagnole est inévitable, parce que les deux ouvrages contribuent à ouvrir en mode collectif, dans le traditionnel format du livre imprimé, le champ de la recherche traductologique, qui est celui de l'histoire de la traduction en 